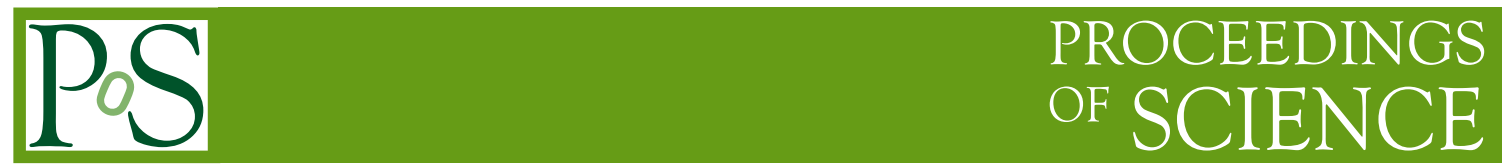

\title{
Performance and calibration of the ATLAS Tile Calorimeter
}

\author{
Antonio Jesús Gómez Delegido ${ }^{a, *}$, on behalf of the ATLAS Collaboration ${ }^{\dagger}$ \\ ${ }^{a}$ Instituro de Física Corpusular, University of Valencia and CSIC, \\ Carrer del Catedratic Jose Beltran Martinez, Paterna, Spain \\ E-mail: antonio.gomez.delegido@cern.ch
}

The Tile Hadronic Calorimeter covers the central region of the ATLAS experiment. Wavelengthshifting fibers carry the light from active plastic scintillator tiles interspersed with steel absorber plates to photomultiplier tubes. Analogue response of the photomultipliers are amplified, shaped, and digitized by a front-end electronics system that samples the signal from about 10000 channels every $25 \mathrm{~ns}$ and stores the data on detector until a trigger decision is received. The dynamic range of each tile covers from $30 \mathrm{MeV}$ to $2 \mathrm{TeV}$. Each step of the process - from collection of scintillation light to signal reconstruction is monitored and calibrated. During LHC Run-2, highmomentum isolated muons and isolated hadrons were used to calibrate the electromagnetic and hadronic response, respectively. The time resolution was studied with multi-jet events. Results of performance studies that address calibration, stability, energy scale, uniformity and time resolution are summarized.

*** The European Physical Society Conference on High Energy Physics (EPS-HEP2021), ***
***26-30 July 2021 ***
*** Online conference, jointly organized by Universität Hamburg and the research center DESY ***

\footnotetext{
* Speaker

$\dagger$ This publication is part of the project RTI2018-094270-B-I00, funded by MCIN/AEI/10.13039/501100011033 and by ERDF A way of making Europe, by the European Union. The speaker acknowledges the grant FPU 2019 funded by MCIN/AEI/ 10.13039/501100011033.
} 


\section{Introduction}

The Tile Calorimeter (TileCal) is the central hadronic calorimeter of the ATLAS experiment [1] at the CERN Large Hadron Collider (LHC) [2]. It plays a crucial role in the measurement of jets and missing transverse energy. TileCal is a sampling calorimeter formed by plastic scintillator tiles as active medium and iron plates as absorbers. It is divided into a central long barrel covering pseudorapidities up to $|\eta|<1.0$ and two extended barrels that provide coverage of the $0.8<|\eta|<1.7$ region, as seen in Figure 1a. These barrels are segmented in 64 modules along the azimuthal direction with equal $\Delta \phi=0.1$ width and have three longitudinal sampling layers (A, $\mathrm{BC}$ and $\mathrm{D})$ to sample the showers at different depths.

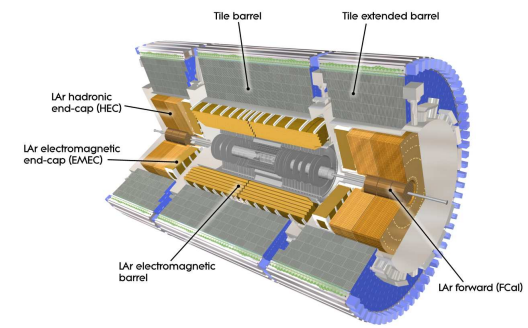

(a)

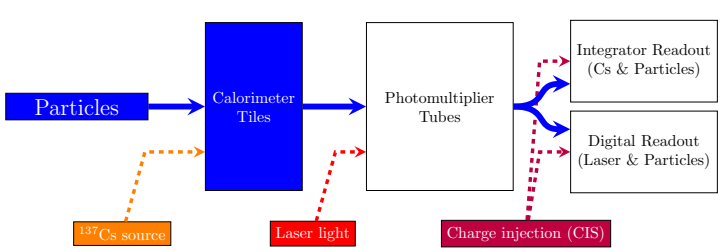

(b)

Figure 1: Cut-away drawing of the ATLAS inner detector and calorimeters (a) [3]. Flow diagram of the readout signal path of the different TileCal calibration systems (b) [3].

When a charged particle goes through the scintillating tiles, ultraviolet light is emitted. This light is collected by wavelength shifting fibers and carried to the photomultipliers (PMT). The PMTs read fibers from multiple tiles, grouped together into cells. Most cells are read out by two PMTs. In total, TileCal has 5182 cells and around 10000 channels. The analog electrical signal produced in each PMT is shaped and amplified in either high or low gain to avoid saturation. The pulses are reconstructed from seven consecutive samples separated by $25 \mathrm{~ns}$. The Optimal Filtering (OF) method is used to reconstruct the amplitude $(A)$ of the pulse, its pedestal and its time.

\section{Calibration systems}

Once the amplitude of a pulse is reconstructed, the energy of the corresponding channel can be evaluated using several calibration coefficients provided by different TileCal calibration systems as follows:

$$
E_{\text {channel }}[\mathrm{GeV}]=A[\mathrm{ADC}] \cdot C_{\mathrm{pC} \rightarrow \mathrm{GeV}} \cdot C_{\mathrm{ADC} \rightarrow \mathrm{pC}} \cdot C_{\mathrm{Cs}} \cdot C_{\mathrm{Laser}} \cdot
$$

The electromagnetic scale calibration constant $C_{\mathrm{pC} \rightarrow \mathrm{GeV}}$, was measured with electrons in dedicated campaigns with beams of known energy. The different calibration systems are used to maintain the time-independent electromagnetic scale [4].

Each of the calibration systems is devoted to control different parts of the readout chain. The flow diagram of the TileCal calibration tools can be seen in Figure 1b. The Cesium System $\left(C_{\mathrm{Cs}}\right)$ calibrates the scintillators and the PMTs responses. The Laser System $\left(C_{\text {Laser }}\right)$ measures gain variations of the PMTs between the cesium calibrations. The Charge Injection System $\left(C_{\mathrm{ADC} \rightarrow \mathrm{pC}}\right)$ calibrates the readout electronics. Finally, the Minimum Bias System monitors the stability of 
the full optical chain. The partial overlap between such systems allows cross-checking and helps to identify problems. The different calibration constants can evolve with time due to variations in the PMT response (due to high-voltage changes or stress due to high light fluxes), scintillator degradation and changes in electronic response.

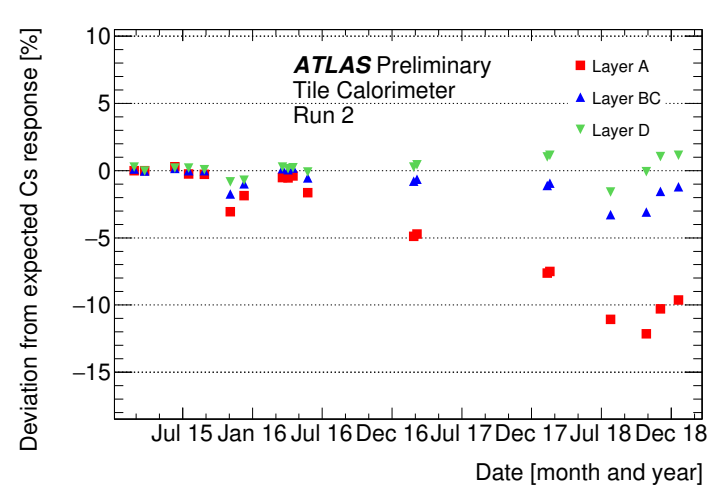

(a)

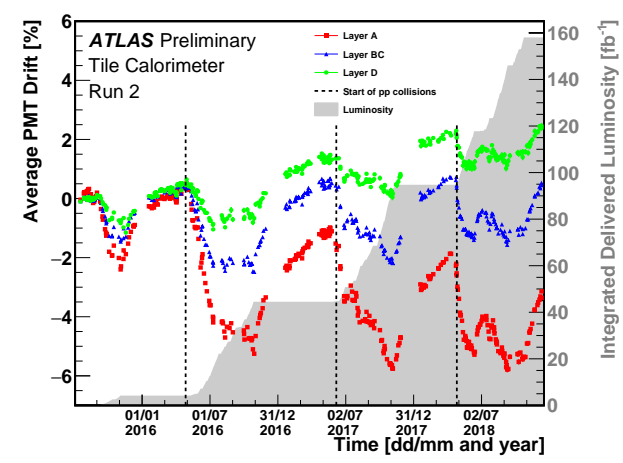

(b)

Figure 2: Time evolution of TileCal cells' mean relative response to Cesium source in three longitudinal layers (a) [3]. Time evolution of the mean relative response of the PMTs in three longitudinal layers measured by the Laser system (b) [3].

The Cesium calibration system employs three ${ }^{137} \mathrm{Cs} \gamma$-sources that are moved with an hydraulic system to illuminate individual cells and calibrate the optical chain (the scintillators and the PMTs). The response of each channel is used to correct deviations from the expected values and maintain the response at the global electromagnetic scale. These corrections are applied through calibration constants. Cesium scans are taken few times per year and the precision of the calibration system is at the level of $0.3 \%$. Figure 2a show the deviation of TileCal response to the ${ }^{137} \mathrm{Cs} \gamma$ - sources with respect to the expected values as a function of time. Layer A exhibits the maximal down-drifts, since it corresponds to the closest cells to the interaction point.

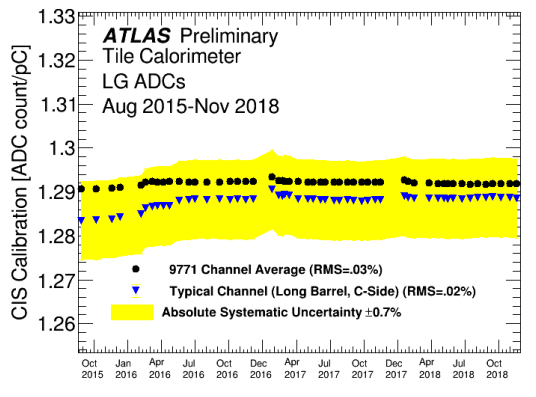

(a)

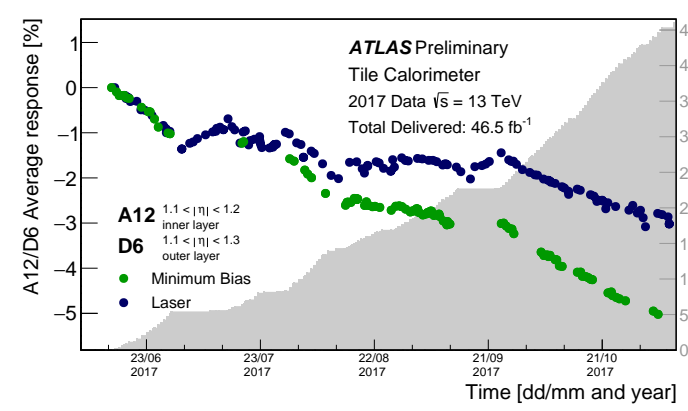

(b)

Figure 3: Detector-wide CIS calibration constant averages of all the low-gain ADCs for a selection of CIS calibration runs (a) [3]. Variation of the response measured by the Minimum Bias and Laser systems for cell A12 as a function of time (b) [3].

The Laser system sends a controlled amount of $532 \mathrm{~nm}$ light onto the PMT photo-cathodes to measure and monitor individual PMT gain variations between Cesium scans. Deviations of the response of the channels with respect to the reference taken at the time of the previous Cesium calibration are translated into additional calibration constants. Laser calibrations are taken weekly 
and the precision of the system is better than $0.5 \%$. The evolution of the mean relative response of the three longitudinal layers measured by the Laser system as a function of time is shown in Figure 2b. The variation of the PMTs response is caused mainly by three competing factors: i) constant up-drift when the PMTs are not operated, ii) down-drifts in high instantaneous luminosity periods and iii) partial recovery of the PMTs during technical stops.

The Charge Injection system (CIS) is used to inject a known charge (between 0 and $800 \mathrm{pC}$ ) in each TileCal channel to calibrate the ADCs response and evaluate linearity. A linear fit yields the $\mathrm{pC} / \mathrm{ADC}$ conversion with a precision of $0.7 \%$ and a stability over time of the order of $0.03 \%$, as can be seen in Figure 3a. Calibration runs are taken with daily to weekly frequency.

Proton-proton collisions are dominated by soft parton interactions. During collisions, the PMTs current due to Minimum Bias (MB) events are integrated during a $10 \mathrm{~ms}$ time window. The response can be used to measure the instantaneous luminosity and monitor the stability of the optical chain. Figure $3 \mathrm{~b}$ shows the response variation of cell A12 with respect to the reference cell D6 as a function of time. The difference between MB and Laser response is interpreted as an effect of scintillator irradiation.

\section{Performance}

The performance of TileCal is monitored online during data-taking. An additional detailed offline assessment is also done within two days after each stable run period, correcting calibration constants if needed. The cells and channels that are identified as problematic and that may affect physics analyses are masked. If the problem is considered to be intolerable, the affected data is not used for physics analyses. TileCal achieved $100 \%$ of data quality efficiency in 2015, 99.3\% in 2016, $99.4 \%$ in 2017 and $100 \%$ in 2018 [5]. Figure 4a shows the evolution of masked cells and channels. Identified problems were mostly fixed in dedicated maintenance campaigns.

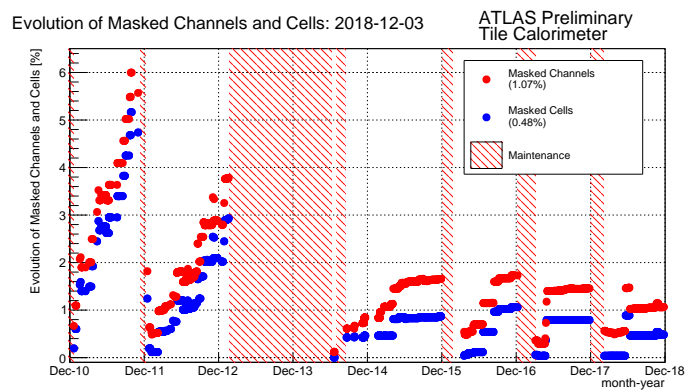

(a)

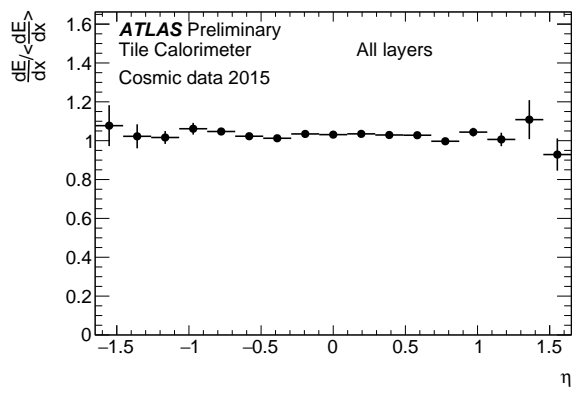

(b)

Figure 4: Evolution of TileCal masked cells (a) [3]. Ratios of the truncated means of the distributions of the energy deposited in the layer cells by cosmic-ray muons per unit of path length $\mathrm{d} E / \mathrm{d} x$, obtained using 2015 data as a function of the pseudorapidity $\eta$ (b) [3].

Cosmic muons are used to study the electromagnetic scale and the cell intercalibration. The energy response non-uniformity across $\eta$ is better than 5\%, as seen in Figure $4 \mathrm{~b}$. TileCal performance was also studied with isolated charge hadrons using the ratio of the reconstructed energy to the track momentum measured by the Inner Detector. This quantity is used to evaluate uniformity and linearity and is expected to be below the unity due to the non-compensating nature of the sampling calorimeter. As is shown in Figure 5a, data and Monte Carlo simulations agree within 5\%. 


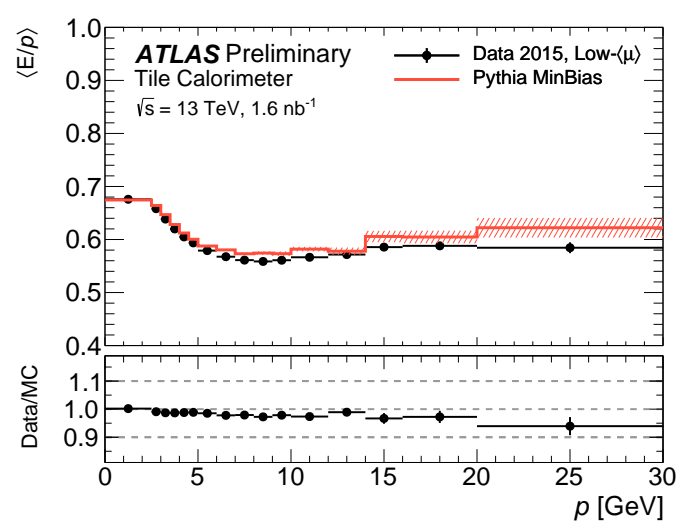

(a)

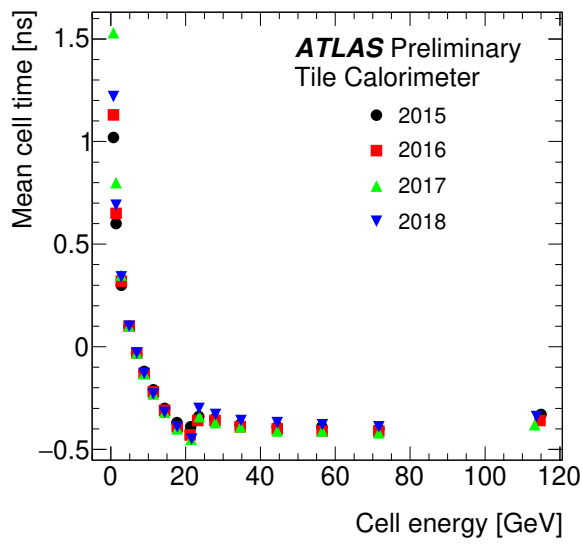

(b)

Figure 5: Calorimeter response to single isolated charged hadrons, characterised by the mean of the energy over momentum $(\mathrm{E} / \mathrm{p})$ as a function of momentum, integrated over the pseudo-rapidity and $\phi$ coverage of the calorimeter (a) [6]. Mean cell time in jet events as a function of the cell energy (b) [6].

The time calibration sets the phase in each channel so that a particle incoming from the interaction point traveling at the speed of light generates a signal pulse peaking at the central sample, which is equivalent to a reconstructed time equal to zero. Laser events and multi-jet events are used to monitor the channel time stability. As it is shown in Figure 5b, the mean cell time measured with jets depends on the deposited energy.

\section{Conclusions}

TileCal plays a crucial role in the ATLAS detector. Several calibration systems guarantee the stability of the cell response and allow to control the readout chain, providing calibrating corrections for the measured variations with respect to the expected response. The performance in TileCal is checked with isolated hadrons and cosmic muons. During Run 2, the data quality efficiency exceeded $99.65 \%$ and the stability of the detector response was better than $1 \%$.

\section{References}

[1] ATLAS Collaboration, The ATLAS Experiment at the CERN Large Hadron Collider, JINST 3 3 (2008) S08003.

[2] ATLAS Collaboration, Readiness of the ATLAS Tile Calorimeter for LHC collisions, Eur.Phys.J.C 70 (2010) 1193 [hep-ex/1007.5423].

[3] ATLAS Collaboration, Approved Tile Calorimeter Plots, 2018. https://twiki.cern.ch/ twiki/bin/view/AtlasPublic/ApprovedPlotsTile.

[4] ATLAS Collaboration, Operation and performance of the ATLAS Tile Calorimeter in Run 1, Eur.Phys.J.C 78 (2018) 12, 987 [hep-ex/1806. 02129].

[5] ATLAS Collaboration, ATLAS data quality operations and performance for 2015-2018 datataking, JINST 15 (2020) 04, P04003 [hep-ex/1911. 04632].

[6] ATLAS Collaboration, Public Tile Calorimeter Plots for Collision Data, 2018. https:// twiki.cern.ch/twiki/bin/view/AtlasPublic/TileCaloPublicResults. 